\title{
OJA/AA \\ AIAA 2001-2159 \\ Laminarization of Turbulent Boundary Layer on Flexible and Rigid Surfaces
}

Lucio Maestrello

NASA Langley Research Center

Hampton, VA

\section{7th AIAA/CEAS Aeroacoustics Conference 28-30 May 2001} Maastricht, The Netherlands

For permission to copy or republish, contact the copyright owner named on the first page.

For AIAA-held copyright, write to AIAA Permissions Department, 1801 Alexander Bell Drive, Suite 500, Reston, VA, 20191-4344. 
AIAA-2001-2159

\title{
LAMINARIZATION OF TURBULENT BOUNDARY LAYER ON FLEXIBLE AND RIGID SURFACES
}

\author{
Lucio Maestrello \\ NASA Langley Research Center \\ Hampton, VA 23681-2199
}

\begin{abstract}
$\underline{\text { Abstract }}$
An investigation of the control of turbulent boundary layer flow over flexible and rigid surfaces downstream of a concave-convex geometry has been made. The concave-convex curvature induces centrifugal forces and a pressure gradient on the growth of the turbulent boundary layer. The favorable gradient is not sufficient to overcome the unfavorable; thus, the net effect is a destabilizing of the flow into Görtler instabilities. This study shows that control of the turbulent boundary layer and structural loading can be successfully achieved by using localized surface heating because the subsequent cooling and geometrical shaping downstream over a favorable pressure gradient is effective in laminarization of the turbulence. Wires embedded in a thermally insulated substrate provide surface heating. The laminarized velocity profile adjusts to a lower Reynolds number, and the structure responds to a lower loading. In the laminarization, the turbulent energy is dissipated by molecular transport by both viscous and conductivity mechanisms. Laminarization reduces spanwise vorticity because of the longitudinal cooling gradient of the sublayer profile. The results demonstrate that the curvature-induced mean pressure gradient enhances the receptivity of the flow to localized surface heating, a potentially viable mechanism to laminarize turbulent boundary layer flow; thus, the flow reduces the response of the flexible structure and the resultant sound radiation.
\end{abstract}

\section{Background}

Turbulent boundary layer control is achievable experimentally by using localized surface heating in a region of pressure gradient. Localized heating leads to an increase in stability and critical Reynolds number. A new velocity profile adjusts to the laminarized Reynolds number, and results in lower skin friction and structural loading. This transition occurs naturally in high speed flow and causes hypersonic flow to be laminar and a hot jet to be quieter than a cold jet. The present experiment is designed to demonstrate flow stability for subsonic boundary layer flow. Laminarization is achieved by the natural cooling of the flow downstream of a heated wire strip placed on a concave surface. The effectiveness of the technique depends on the pressure gradient, freestream conductivity, diffusivity, temperature, and Reynolds number. The coupling between heat flux and streamwise pressure gradient influences the stability sufficiently to reverse the state of the flow from turbulent to laminar.

The shear flow over a concave surface is subject to centrifugal instability whose inviscid mechanism was given first by Rayleigh ${ }^{1}$ (1916) and Reynolds ${ }^{2}$ (1884) and in recent works by Narasimha and Sreenivasan; ${ }^{3}$ Hoffmann, Muck, and Bradshaw; ${ }^{4}$ Hall; ${ }^{5}$ Floryan; ${ }^{6}$ Maestrello and El-Hady; ${ }^{7}$ and Bayliss et al. ${ }^{8}$ These works have indicated that the flow over a concave surface is potentially unstable, resulting in two- and three-dimensional disturbances, whereas the flow over a convex surface is stabilizing. The overall effect of the surface curvature on the flow cannot be predicted a priori; it depends on the parameters of the flow and initial disturbance.

In the last decade, the study of nonlinear and chaos control has attracted much attention. ${ }^{9-15}$ Recently, investigations have shifted to spatiotemporal systems to control pattern formation including turbulence. Turbulence remains an extremely important problem in the science of nature and is an example of spatiotemporal chaos. The deterministic and chaotic responses need to be distinguished from stochastic behavior. Typically, random behavior can arise in a number of ways, but actually, the behavior is the result of deterministic 
chaos that appears random because of the lack of sufficient information about initial starting condition. $9,10,14$ Vibration control using an actuator for stabilizing panel vibration satisfying a nonlinear beam equation is studied by Chow and Maestrello ${ }^{16}$ with a perturbation technique. The vibration control principle can also be applied to other problems such as nonlinear wave propagation and flow stability and control. A potentially less restricted method of control is by passive surface heating; 17 Maestrello and Ting ${ }^{12}$ analyzed this problem by using the method of matched asymptotes as a "triple-deck" problem. This analysis confirmed that a small amount of localized surface heating can excite local disturbances which increase the momentum near the wall and reduce the displacement thickness and, as a result of downstream cooling, laminarize the turbulence of the flow.

In this paper, the effect of the curvature-induced pressure gradient on the growth of the turbulent boundary layer is studied. In particular, with the use of a wire strip on the concave portion of the surface, the behavior resulting from an imposed steady localized heating is investigated. The boundary layer is turbulent, nearly two-dimensional in the mean; three-dimensional centrifugal instability is created by the largest eddies present in the flow approaching the concave portion of the curvature. Centrifugal effects can be categorized into three types: (1) change in the turbulent structure induced by the wall curvature, (2) generation of longitudinal vortices, and (3) effect of the longitudinal vortices on turbulent structure. Over the concave portion of the surface, vortex cells are triggered by the interaction between the centrifugal instability and the level of fluctuations created by the eddies in the boundary layer.

Structural vibration and resultant sound radiation can also be controlled by suppressing waves on the structure rather than by controlling the boundary layer itself. One method uses so-called "rubber wedges" designed to attenuate waves incident and reflecting from the boundaries. Laboratory testing at low speed flow and flight testing on a Boeing 727 airplane at Mach number 0.85 and altitude of 31000 feet show $15 \mathrm{~dB}$ and $8 \mathrm{~dB}$, respectively, of broadband acoustic power reduction. The added mass for the modified boundaries was approximately 30 percent of the panel weight. ${ }^{18,19}$

The analysis begins with passive control by localized surface heating, and the experiment begins with measurements of local fluctuations and mean velocities, followed by the distribution of the average temperature cooling downstream of the heating wire, the sequence of Görtler vortices during laminarization stages, and finally the fluctuations and mean velocity of the uncontrolled and the controlled boundary layer. The analysis of control by surface heating is described in Sec. 2. Sec. 3 describes the configuration geometry and instrumentation. Results on the control of Görtler instability and laminarization of the boundary layer and structural response are described in Sec. 4.1 and Sec. 4.2.

\section{Flow Analysis of Localized Surface Heating and Self Downstream Cooling}

Localized surface heating in air alters the growth of the flow instabilities by subsequent cooling downstream. As a result, the flow stability is increased by the modifications of the velocity and pressure distributions. The analysis must begin with the energy equation, even for incompressible flow. The problem deals with a change in thermal boundary conditions which, in turn, creates a disturbance field in the boundary layer. A heuristic argument was presented to explain the equivalence between heat flux and effective normal velocity at the wall, since the coupling between the thermal and mechanical effects is provided by the dependence of viscosity on temperature. ${ }^{12}$ Qualitative effects on the stabilization of the boundary layer due to localized heating with subsequent downstream cooling were obtained for air because the viscosity $\mu$ increases with temperature $T$; that is

$$
d \mu / d T>0
$$

Surface heating with subsequent downstream cooling changes the velocity profile due to the dependence of viscosity on temperature. An extra term $(d \mu / d T)$ $(\partial T / \partial y)(\partial u / \partial y)$ appears on the right-hand side of the momentum equation for an incompressible boundary layer where $\mathrm{y}$ is the coordinate normal to the surface. Here $\mu, T, u$, and $y$ are nondimensional quantities, and $u$ is the velocity. In the experiment described in this paper, a concave-convex surface is used to laminarize the turbulent boundary layer. A thin nickel-chromium wire strip embedded in the concave part of the curved surface is heated by a steady electric current. Local heating and subsequent downstream cooling changes the velocity profile and pressure distribution where the surface geometry changes from a concave to a convex surface and then to a flat region. The favorable effect can be 
assessed where the temperature reaches an ambient value over the flat region. It is well-known that such a sudden local change of the boundary condition creates a disturbed flow field in the boundary near the heating strip. The present problem differs from that in work done by Stewartson ${ }^{20}$ and Smith ${ }^{21}$ because of a sudden change in the thermal boundary condition as described by Liepmann, Brown, and Nosenchuck. ${ }^{17}$

The analysis begins with the energy equation for an incompressible flow. The governing equations in dimensionless quantities for $u^{(2)}(x, y, t), v^{(4)}(x, y, t)$, $T^{(1)}(x, y, t)$, and $p^{(3)}(x, t)$, corresponding to velocities $u$ and $v$, temperature $T$, and pressure $p$ for the lower deck are shown as follows with the superscripts indicating the order of the perturbation in the expansion schemes and identifying the corresponding power of $\varepsilon$ :

$$
\begin{gathered}
u_{x}^{(2)}+v_{y}^{(4)}=0 \\
u_{t}^{(2)}+\beta y u_{x}^{(2)}+\beta v_{y}^{(4)}=-p_{x}^{(3)}(x, t)+\mu_{y y}^{(2)}+\Lambda \beta T_{y}^{(1)} \\
T_{i}^{(1)}+\beta y T_{x}^{(1)}=(\operatorname{Pr})^{-1} T_{y y}^{(1)} \\
p_{x}^{(3)}(x, t)=\frac{1}{\pi} \int_{-\infty}^{\infty} \frac{A_{\xi}(\xi, t) d \xi}{x-\xi}
\end{gathered}
$$

where $\Lambda$ is equal to $d \ln (v) / d \ln (T), \operatorname{Pr}$ is the Prandtl number at $T_{0}$, and $\beta$ is the slope of the velocity profile at the wall. The variation of viscosity with temperature in the momentum equation, because it is associated with pressure gradient, becomes the mechanism that effectively alters the turbulent boundary layer. For the Blasius profile, $\beta$ equals 0.33206 . Notice the $\Lambda \beta T_{y}^{(1)}$ dependency of $u$ and $T$ due to the forcing term for $u^{(2)}, v^{(4)}$, and $p^{(3)}$. The term $A_{\xi}(x, t)$ is given by Hilbert transforms, ${ }^{12}$ and

$$
A(x, t)=\beta^{-1} u^{(2)}(x, y \rightarrow \infty, t)
$$

On the heating wire, it is assumed that

$$
T^{(1)}=\frac{T_{w}-T_{0}}{\varepsilon T_{0}}=1+\cos \omega t
$$

This analysis provides the solution of the velocity and pressure distributions. If the frequency is finite, $O(1)$, the full unsteady analysis has to be carried out. In the usual linearized incompressible equations, the unsteady term and the last term in equation (2) are absent; the energy equation for temperature is not needed for the solution of the velocity disturbances. An example of the pressure distribution $p^{(3)}=-\left(\Delta p / p U_{0}^{2} \varepsilon^{3}\right)$ is plotted in Fig. 1 with $\Delta x=0.75$ and $\Delta T=100^{\circ} \mathrm{C}$ ( $\Delta T$ is the temperature of the heating strip above the plate temperature). For $x<0$, the pressure distribution decreases slightly; for $x>0$, increases drastically over the heating strip to reach a maximum at the end of the strip and decreases drastically downstream. Heating of the air destabilizes over the heating strip because $d \mu / d T$ is greater than zero, but it stabilizes downstream of the heating strip. The overall values for temperature change $\Delta T$, Prandtl number $\operatorname{Pr}$, and $\Lambda$ are given in Table 1 . The contribution of the steady-quasisteady terms depends on the size of $\Delta T$ and $d \mu / d T$, whereas the unsteady terms depend on frequencies and phases.

Table 1. Values for Temperature Change $\Delta T$, Prandtl Number $P r$, and $\Lambda$

\begin{tabular}{|c|c|c|}
\hline$\Delta T,{ }^{\circ} \mathrm{C}$ & $\operatorname{Pr}$ & $\Lambda$ \\
\hline 10 & 0.72 & 1.535 \\
100 & 0.72 & 16.52 \\
\hline
\end{tabular}

\section{Configuration Geometry and Instrumentation}

The experiment is conducted in an open-circuit wind tunnel with a $38.1-$ by $38.1-\mathrm{cm}$ test section at a speed of $36.6 \mathrm{~m} / \mathrm{s}$ on a plate $4.8 \mathrm{~m}$ long. (See Fig. 2.) A portion of the plate is concave and a portion is convex with flat regions upstream and downstream (Fig. 2). Upstream, the plate features an elliptic leading edge with a thickness of $2.54 \mathrm{~cm}$, and downstream it features a trailing edge with controllable angle flap. The concave-convex portion is described by a seventh-degree polynomial with the first three derivatives continuous at both ends. This degree of smoothness is designed to prevent singularities from being generated at the points where the surface becomes flat. This geometry was selected to illustrate the effect of curvature-induced pressure gradient flow and flexible structure stability. The concave portion has an adverse pressure gradient and the mean flow decelerates, whereas downstream the curvature becomes convex and produces a favorable pressure gradient and the flow accelerates. 
The surface contains a thermally insulated substrate (Space Shuttle tile). The flow behaviors caused by imposed steady heating from two wires in the concave portion were investigated. The wires are nickel-chromium strips with resistance about $4 \Omega / \mathrm{m}$. The wires are held in position under tension during the heating and cooling cycles. The power supply operates in a direct-current mode in a manner similar to that of a previous experiment using leading edge heating on a flat plate. ${ }^{13}$ The heater current and voltage are continuously monitored, and they are used to determine the total power input during the control cycle. The surface temperature downstream of the heating wires is measured by a line of thermocouples placed along the sidewall corner of the tunnel test section. Downstream of the concaveconvex curvature, the surface is flat, and a flexible aluminum plate is located $40 \mathrm{~cm}$ from the curvature. The plate, 45 by 20.3 by $0.08 \mathrm{~cm}$, with clamped edges is mounted flush with the surface and is used to study the structural loading. The rest of the surface is rigid. A hot-wire probe, accelerometer, wall pressure transducer, temperature sensors, and infrared thermography ${ }^{22}$ were used to evaluate the flow and structure response. The infrared thermography technique is a nonintrusive method to investigate the changes of the Görtler vortices with temperature gradient. The wind tunnel geometry, flow quality, and background noise permits a study of the laminarization control problem of a turbulent boundary layer at low speed.

\section{Experimental Results}

The mean velocity profiles, perturbation velocity, and the infrared thermogram of the developing vortices over the concave-convex surface are evaluated. The uncontrolled boundary layer is turbulent upstream and downstream of the curvatures. The concave curvature has a destabilizing effect because it increases the levels of Reynolds shear stress and turbulent energy and enhances the turbulent mixing, whereas the convex curvature has a stabilizing effect on Reynolds shear stresses and turbulent energy level; turbulent mixing has a decreasing level compared with an equivalent straight shear layer flow.

\subsection{Görtler Instability, Perturbation Velocity. Mean Velocity, and Laminarization of Turbulence}

The perturbation velocity $u(x, t)$ versus $t$, the mean velocity profile $y(x) / \theta(x)$ versus $u(x) / U_{o}$, and the frictional velocity $u(x) / u_{\tau}(x)$ versus $y u_{\tau}(x) / v$ at location $x_{1}$ are shown in Fig. 3, where $u(x, t)$ is the local fluctuation velocity, $y$ is the coordinate, $\theta$ is boundary layer displacement thickness, $u$ is mean velocity, $U_{o}$ is freestream velocity, $u_{\tau}$ is frictional velocity, and $v$ is kinematic viscosity. The location of $x_{1}$ is $40 \mathrm{~cm}$ upstream of the curvature. A wind tunnel condition is chosen from the calibration runs based on flow, vibration, and noise quality. From the mean velocity profiles and perturbation velocity, one can deduce that the uncontrolled boundary layer is turbulent at freestream velocity, $U_{o}=36.6 \mathrm{~m} / \mathrm{sec}$, and Reynolds number, $R_{\theta}\left(x_{1}\right)=2010$. The frictional velocity profiles are comparable with standard wall law, flat plate, turbulent boundary layer.

The choice of the geometry in Fig. 2 permits laminarization of the boundary layer downstream of a concave-convex curvature by localized heating on the concave portion of the surface, whereas the flow remains intermittently turbulent upstream. The average surface temperature distribution $T / T_{\text {ref }}$ downstream of the heating wires decays exponentially with distance to cause the flow to laminarize $\left(T_{\text {ref }}\right.$ is the ambient temperature of the wind tunnel surface). (See Fig. 4.) Figures 5(a) to $5(f)$ show the infrared thermogram vorticity patterns over the concave-convex curvature. The Görtler vortices originate from the concave portion of the surface, and they become stabilized over the convex portion of the surface as the temperature decays with distance. Stability of the vortices is indicated by the increase in wavelength and a sudden increase in size as the number of vortices reduces. The temporal stages toward laminarization are related to the thermoconductivity gradient of the surface and flow characteristics. Distinct features in the time sequences of the vorticity pattern are shown in each step. (See Fig. 5.) During control stages, the heat flux through the wire is gradually increased until the reversion from a turbulent to a laminar state is established. Then the amount of heat flux is reduced by 25 percent equivalent to $200 \mathrm{~W}$ after establishing laminarization of the turbulent boundary layer. The analysis in Sec. 2 can provide the guidelines. The Görtler patterns stretch downstream over the convex portion with increased stability as the temperature decreases with distance (Fig. 5(a)). The perturbations of the flow over the heating wires amplify a region of unfavorable pressure gradient, and then the perturbation decays due to cooling in the region of favorable gradient. This reversion in the amplification is due to both geometrical shaping of the curvature profile and surface cooling with downstream distance. The figure also indicates that 
the changing pattern is not two-dimensional. In Figs. 5(b), 5(c), and 5(d), the temperature of the wire increases (red indicates the higher and blue the lower temperature). The decrease in wall temperature with downstream distance causes drastic changes in vorticity; the boundary layer laminarizes in the process. In the laminarized state only two large vortices remain, the spatial change is an indication of stabilization (Figs. 5(e) and 5(f)). The vorticity is concentrated in the core surrounded by a large-scale motion with small-scale vortices embedded within; it is possible to recognize successive changes as the wire temperature increases.

The temporal sequences of the velocity perturbations, prior, during, and after laminarization, are shown in Fig. 6, at $x_{2}$ located $40 \mathrm{~cm}$ downstream of the flexible panel. Six successive time step intervals $\Delta t$ are shown, from the turbulent state, time step 1 , to laminar state, time step 6 . The features seen during transition are like the features seen in a turbulent spot, that is, time step 5 . The perturbations in time steps 2,3 , and 4 have a higher amplitude than in the turbulence in step 1 and should be interpreted as the envelop of all the possible types of disturbance amplification. The process of laminarization also indicates a change in Reynolds numbers from $R_{\theta}\left(x_{2}\right)=2728$ in turbulent state to $R_{\theta}\left(x_{2}\right)=983$ in laminarized state. These changes in perturbation velocity and mean velocity profiles are shown in Fig. 7. The mean profile (Fig. 7(c)) changes from turbulent in the initial stage, to laminarized in the final stage resembling the Blasius profile. The perturbation velocity changes also indicate laminarization of the turbulence. During the control stages, as heat on the wire strips increases, the boundary layer at $x_{1}$ becomes intermittently turbulent with an oscillation in $R_{\theta}\left(x_{1}\right)$ between 5 to 15 percent from its original value.

The pressure gradient in the control region of the heating wires and downstream is essential in order to maintain the coupling between the thermal gradient and sublayer because coupling heat input with the flow at zero pressure gradient is virtually impossible. This important feature "pressure gradient" is indicated on the right-hand side of the momentum equation (eq. (2)) with viscosity as a function of temperature. The rate of cooling with distance is the stabilizing mechanism; as a result, the critical Reynolds number increases. There is no bounded limit on how far laminarization can be extended because the stability increases with the increasing ratio between local heat flux and thermal conductivity and temperature. The whole laminarization process looks like transition to turbulence in reverse as it can be observed from a fixed point.

\subsection{Panel Structure Response and Control}

The response of the panel is induced by the convecting boundary layer loading, both turbulent and laminar. The static pressure difference across the panel is $2.5 \mathrm{~kg} / \mathrm{m}^{2}$, the static deflection is less than the panel thickness. The pressure difference has an effect on the structural response. The response is measured by an accelerometer at the panel center.

The normalized power spectral density $G(f)$ of the acceleration for the turbulent and the laminarized boundary layer loading is shown in Fig. 8. No attempt is made to measure the spatiotemporal response. The spectrum is dominated by the lower frequencies, an expected result for low speed boundary layer loading. The controlled spectrum has a lower amplitude than the uncontrolled one; their differences increase with frequency (Fig. 8). At the low frequencies, the reduction in power is approximately $7 \mathrm{~dB}$; at high frequencies, more than $10 \mathrm{~dB}$. Laminar boundary layer loading reduces the structural response with respect to the turbulent boundary layer. The acoustic background noise of the facility did not permit measurement of the acoustic power (radiated) by the panel. From the response, one expects that the acoustic spectrum will be dominated by the low frequencies with a lower level for the laminarized boundary layer loading than for the turbulent one. Larger differences in structural response are expected for higher speed flow, since the convected waves of the panel dominate the response over a wider bandwidth.

\section{$\underline{5 . \text { Conclusions }}$}

An investigation of the control of the turbulent boundary layer over rigid and flexible surfaces downstream of a concave-convex geometry was made. Heating was applied at the concave surface. Pressure gradient forces crucially influence the control of turbulence. The Gortler spatial pattern that extends over the curvature is marked by changes of vorticity over time. The physical significance is that the cooling pattern over a favorable gradient is a gain in stability of the flow. Laminarization reduces the Reynolds number, spanwise vorticity, wall pressure fluctuations, and structural loading. The 
effectiveness of the technique depends on pressure gradient, freestream conductivity, diffusivity, temperature, and Reynolds number. The coupling between heat flux and streamwise pressure gradient influences the stability sufficiently to reverse the state of the flow. The drawback of using such a control system is that the power required can be higher than the power reduction in the system response. Potential applications include aircraft and ground vehicles where concave-convex surfaces are typically found on the windshield region as well as on a flat surface where a local pressure gradient is induced.

\section{References}

1. "413. On the Dynamics of Revolving Fluids," Scientific Papers by Lord Raleigh, Vol. VI: 1911-1919, Dover Publications, New York, 1964. (Originally published in Proceedings of Royal Society, A, XCIII, 1916, pp. 148-154.)

2. Reynolds, O., "The Two Manners of Motion of Water," Proceedings of Royal Institute of Great Britain, 11, 1884.

3. Narasimha, R., and Sreenivasan, K. R., "Relaminarization of Fluid Flows," Advances in Applied Mechanics, 19, 1979, pp. 221-308.

4. Hoffmann, P. H., Muck, K. C., and Bradshaw, P., "The Effect of Concave Surface Curvature on Turbulent Boundary Layers," Journal of Fluid Mechanics, 161, 1985, pp. 371-403.

5. Hall, P., "The Nonlinear Development of Görtler Vortices in Growing Boundary Layer," Journal of Fluid Mechanics, 193, 1988, pp. 243-266.

6. Floryan, J.M., "On the Görtler Instability of Boundary Layer," Progress in Aerospace Sciences, 28, 1991, pp. 235-271.

7. Maestrello, L., and El-Hady, N. M., "Radiation and Control of Sound From Evolving Wave Packet on a Concave-Convex Surface," AlAA Journal, 29, 1991, pp. 880-887.

8. Bayliss, A., Maestrello, L., Parikh, E., amd Turkel, E., "Numerical Simulation of BoundaryLayer Excitation by Surface Heating/Cooling," AIAA Journal, 24, 1986, pp. 1095-1101.

9. Ott, E., Grebogi, C., and Yorke, J. A., "Controlling Chaos," Physical Review Letters, 64, 1990, pp. 1196-1199.
10. Pecora, Louis M., and Carroll, Thomas L., "Driving Systems With Chaotic Signals," Physics Review A, 44, 1991, pp. 2374-2383.

11. Ditto, W. L., Spano, M. L., and Lindner, J. F., "Technique for the Control of Chaos," Physica D, 86, 1955, pp. 198-211.

12. Maestrello, L., and Ting, L., "Analysis of Active Control by Surface Heating," ALAA Journal, 23, 1985, pp. 1038-1045.

13. Maestrello, L., and Grosveld, F. W., "Transition Control of Instability Waves Over an Acoustically Excited Surface," AIAA Journal, 30, 1992, pp. 665-670.

14. Maestrello, L., "Controlling Vibrational Chaos of a Curved Structure," ALAA Journal, 2001. (In press)

15. Maestrello, L., "Active Control by Conservation of Energy Concept," AIAA Paper 2000-2045, June 2000.

16. Chow, P. L., and Maestrello, L., "Vibrational Control of a Non-Linear Elastic Panel," International Journal of Nonlinear Mechanics, 2001. (In press)

17. Liepmann, H., Brown, G. L., and Nosenchuck, D. M., "Control of Laminar-Instability Waves Using a New Technique," Journal of Fluid Mechanics, 118, 1982, pp. 187-200.

18. Maestrello, L., "Use of Turbulent Model To Calculate the Vibration and Radiation Responses of a Panel With Practical Suggestions of Reducing Sound Level." Joumal of Sound and Vibration, 5, 1967, pp. 407-448.

19. Baht, W. V., and Wilby, J. F., "Interior Noise Radiation by an Airplane Fuselage Subjected to Turbulent Boundary Layer Excitation and Evaluation of Noise Reduction Treatments." Journal of Sound and Vibration, 18, 1971, pp. 449-464.

20. Stewartson, K., "Multistructured Boundary Layer on Flat Plate and Related Bodies," Advances in Applied Mechanics, 14, 1974, pp. 145-239.

21. Smith, F. T., "On the High Reynolds Number Theory of Laminar Flow," IMA Journal of Applied Mathematics, 28, 1982, pp. 207-281.

22. Carlomagno, G. M., and De Luca, L., "Infrared Thermography Applications in Convective Heat Transfer," Flow Visualization V, edited by $\mathrm{R}$. Reznicek, Hemisphere Publishing Corporation, New York, 1990, pp. 843-848. 


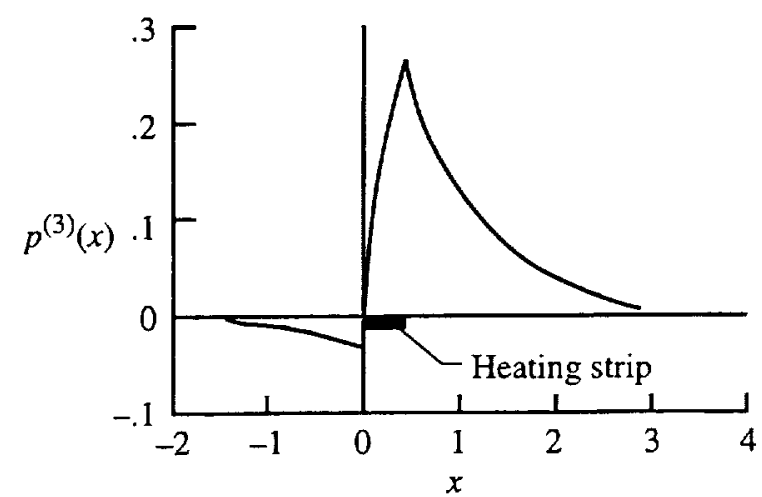

Figure 1. Pressure distributions induced by steady surface heating.

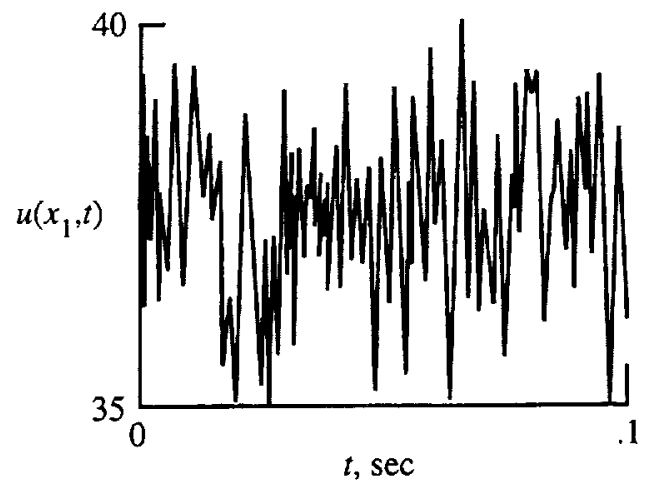

a) Velocity fluctuation.

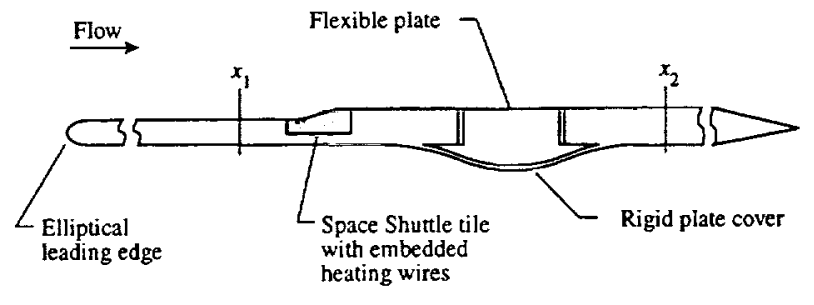

Figure 2. Experimental model.

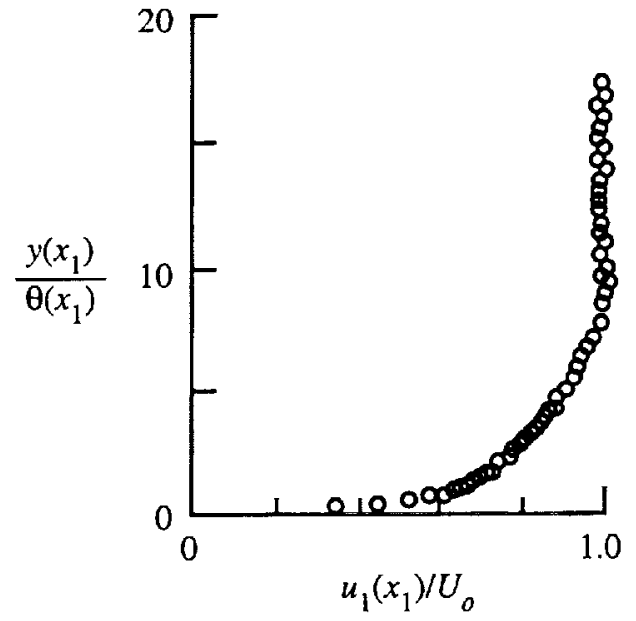

b) Mean velocity profile.

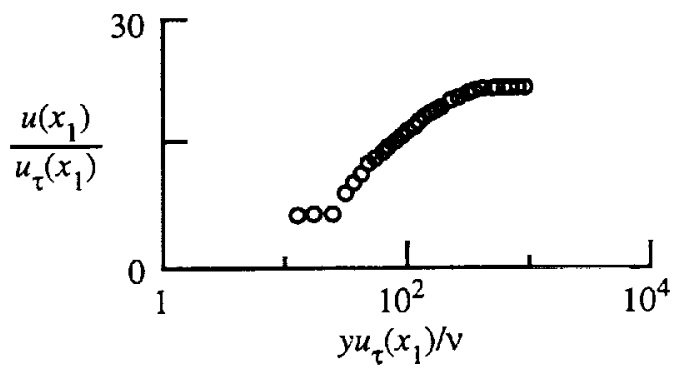

c) Frictional velocity profile.

Figure 3. Turbulent boundary layer at location $x_{1}$. 


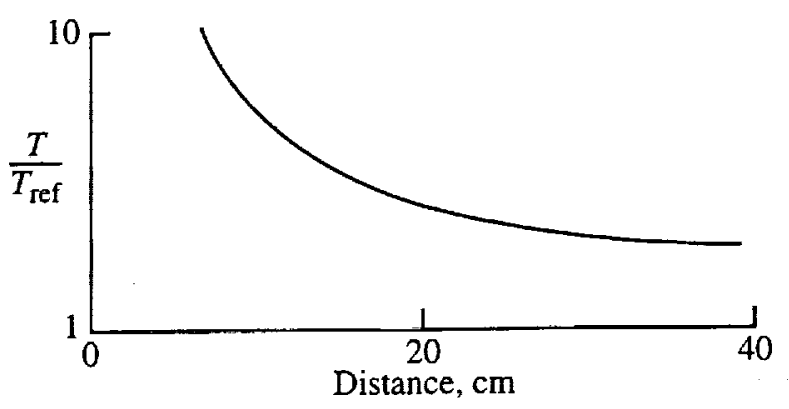

Figure 4. Average surface temperature downstream of heating. 


\section{Upstream}
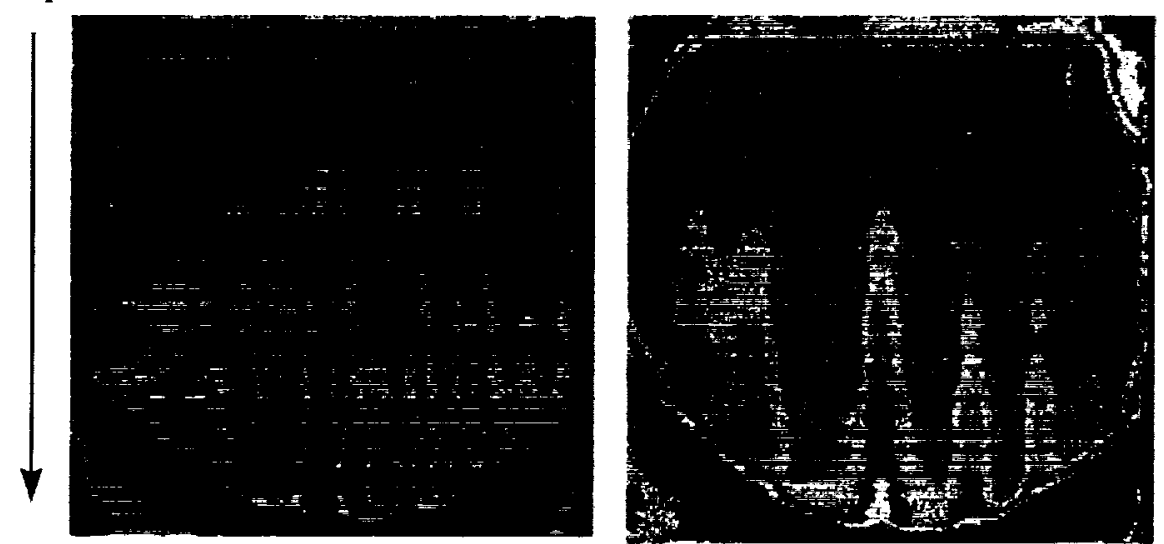

Downstream

a) Turbulent vortices.

b) Turbulent vortices.

\section{Upstream}
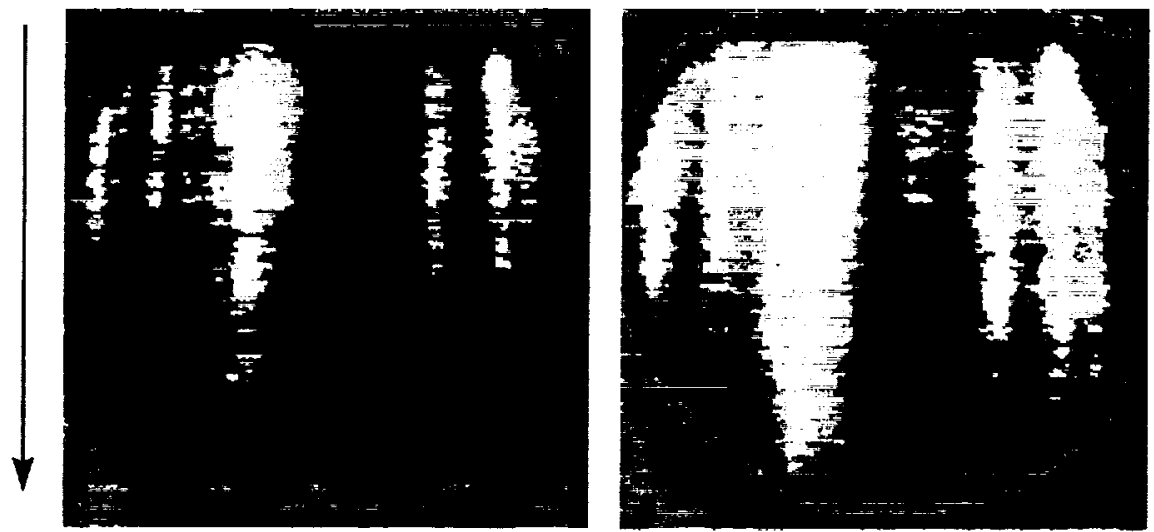

Downstream

c) Turbulent vortices.

d) Transitional vortices.
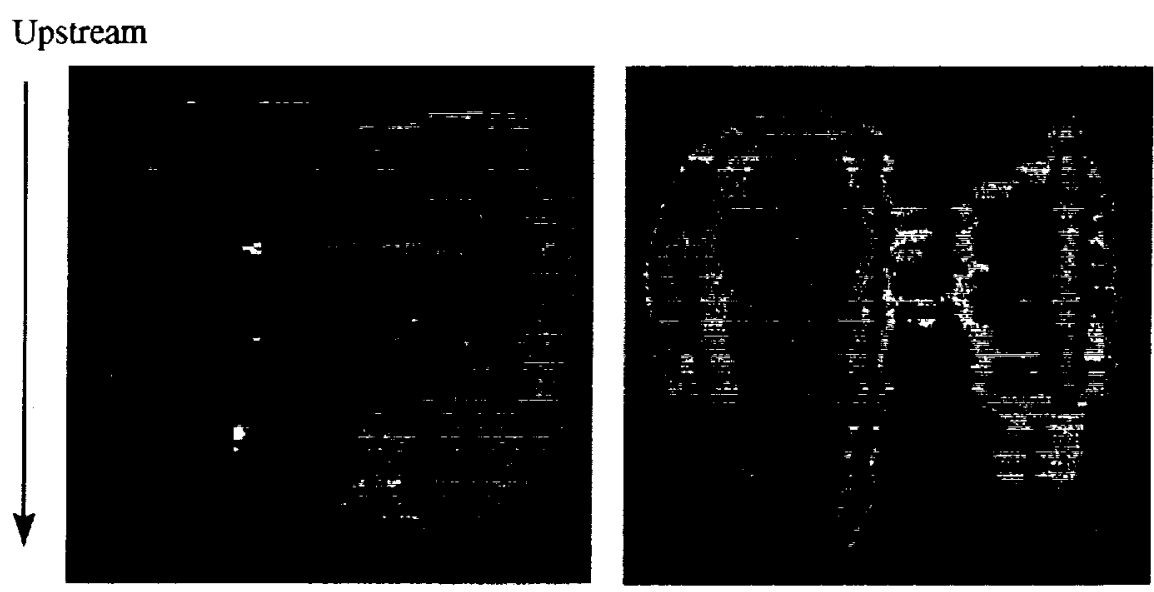

Downstream
e) Laminarized vortices.
f) Laminarized vortices.

Figure 5. Time sequence of Görtler vortices over concave-convex curvature during laminarization stages. 


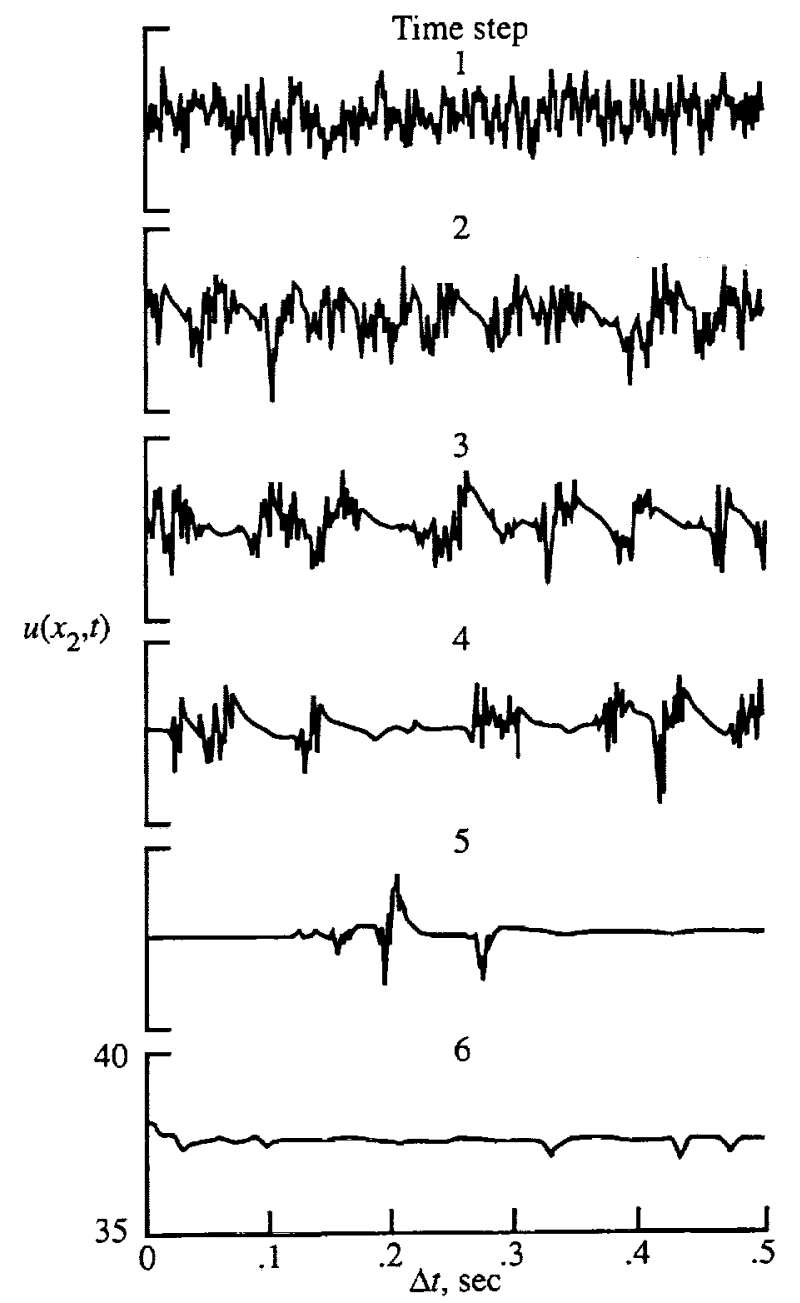

Figure 6. Fluctuation velocity for successive time steps $\Delta t$ from turbulent to laminarized state at location $x_{2}$. 


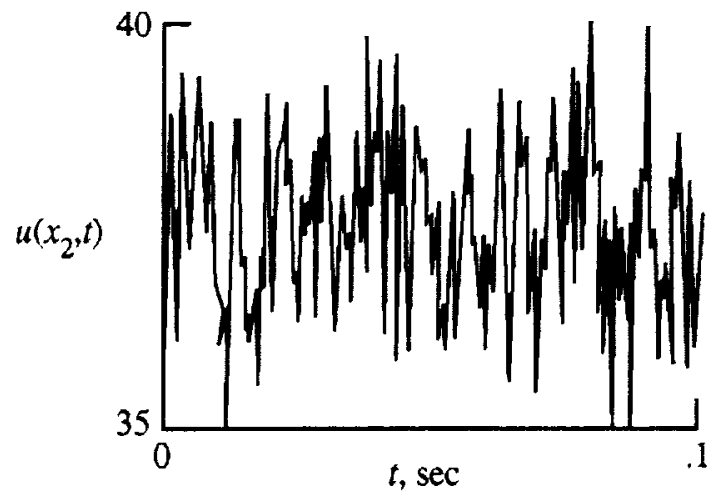

a) Uncontrolled velocity fluctuation.

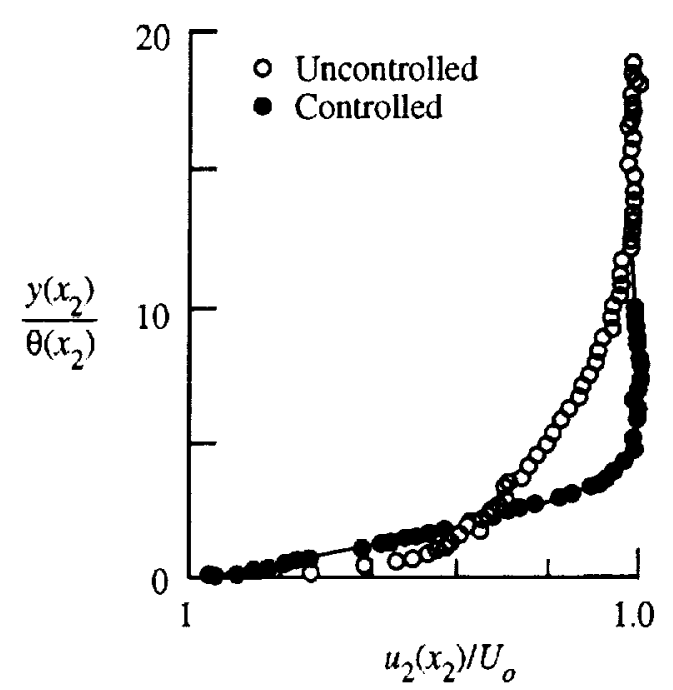

c) Mean velocity profile.

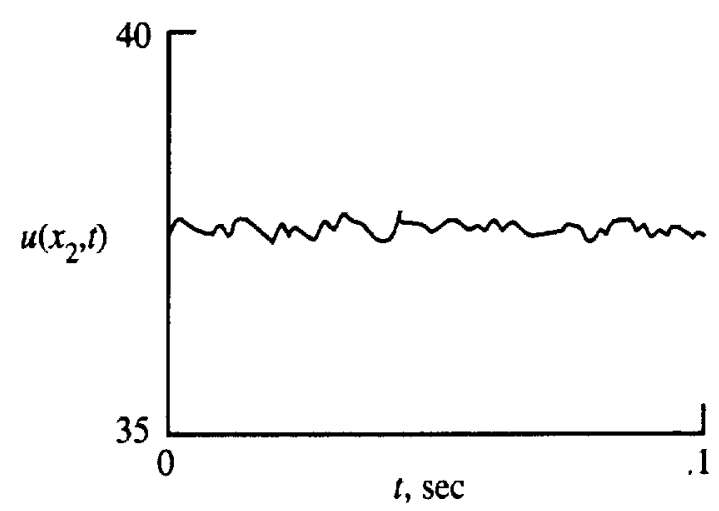

b) Controlled velocity fluctuation.

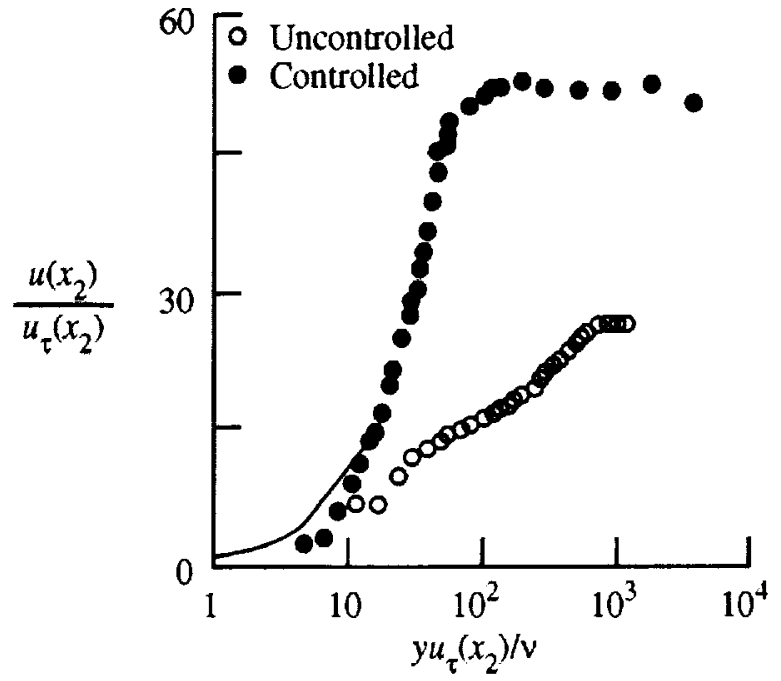

d) Frictional velocity profile.

Figure 7. Turbulent and laminarized boundary layer at location $x_{2}$.

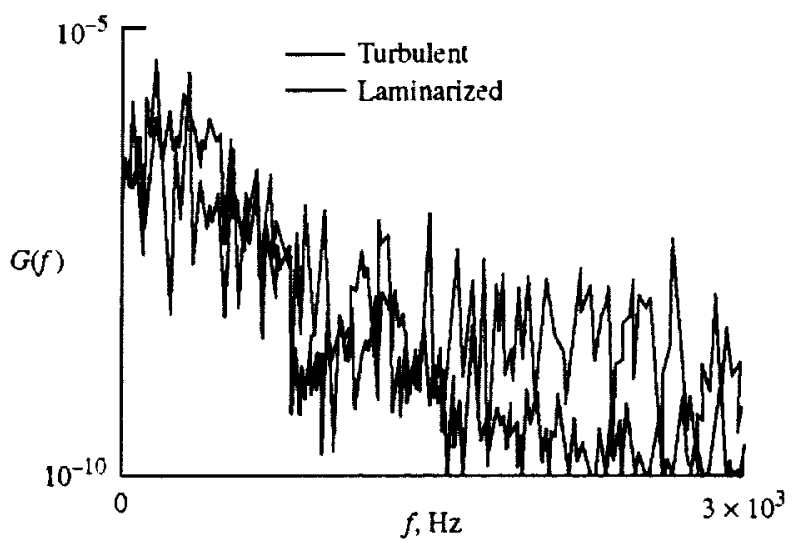

Figure 8. Response of the panel structure forced by turbulent and laminarized boundary layers. 
